Article

\title{
Target Channel Visiting Order Design Using Particle Swarm Optimization for Spectrum Handoff in Cognitive Radio Networks
}

\section{Shilian Zheng ${ }^{1,2, *}$, Zhijin Zhao ${ }^{3}$, Changlin Luo ${ }^{4}$ and Xiaoniu Yang ${ }^{1,2}$}

1 Science and Technology on Communication Information Security Control Laboratory, Jiaxing 314033, China; E-Mail: xiaonyang@126.com

2 School of Telecommunications Engineering, Xidian University, Xi'an 710071, China

3 School of Telecommunications Engineering, Hangzhou Dianzi University, Hangzhou 310018, China; E-Mail: zhaozj03@hdu.edu.cn

4 Nanhu School, Jiaxing University, Jiaxing 314001, China; E-Mail: luochanglin@gmail.com

* Author to whom correspondence should be addressed; E-Mail: lianshizheng@126.com;

Tel.: +86-573-8367-8066.

Received: 29 March 2014; in revised form: 29 July 2014 / Accepted: 8 August 2014 /

Published: 18 August 2014

\begin{abstract}
In a dynamic spectrum access network, when a primary user (licensed user) reappears on the current channel, cognitive radios (CRs) need to vacate the channel and reestablish a communications link on some other channel to avoid interference to primary users, resulting in spectrum handoff. This paper studies the problem of designing target channel visiting order for spectrum handoff to minimize expected spectrum handoff delay. A particle swarm optimization (PSO) based algorithm is proposed to solve the problem. Simulation results show that the proposed algorithm performs far better than random target channel visiting scheme. The solutions obtained by PSO are very close to the optimal solution which further validates the effectiveness of the proposed method.
\end{abstract}

Keywords: particle swarm optimization; cognitive radio; spectrum handoff; target channel

\section{Introduction}

Spectrum is generally regulated by governments via a command and control approach. However, this approach has led to underutilization of a spectrum in vast temporal and geographic dimensions as 
shown in the survey made by the Federal Communications Commission (FCC) [1]. Motivated by this observation, cognitive radio (CR) has been proposed to improve spectrum utilization by dynamically accessing spectrum "white spaces" (i.e., spectrum holes) without causing harmful interference to primary users [2]. In this dynamic spectrum access framework, CRs are considered as secondary users (SUs) of the spectrum. When a primary user (licensed user) reappears on the current channel, the CRs need to vacate the channel and reestablish a communications link on some other channel to avoid interference to primary users. This process is referred to as spectrum handoff [3]. Target channels play an important role in spectrum handoff design because target channels are the hopes that SUs can resume their unfinished transmissions.

Based on the decision timing for selecting target channels for spectrum handoff, the target channel selection approaches can be categorized into two kinds: on-demand channel selection for reactive-decision spectrum handoff and predetermined channel selection for proactive-decision spectrum handoff [4]. On-demand channel selection searches target channels after spectrum handoff [5-7]. This may result in long spectrum handoff delay because an available channel needs to be searched in a wide frequency range. Predetermined channel selection determines target channels before spectrum handoff based on the long-term history information on channel status. It can save spectrum sensing time. This paper focuses on predetermined channel selection. Most prior work on predetermined channel selection selected a single target channel based on some objective, for instance, maximum spectrum lifetime [8], maximum residual idle time [9], maximum idle probability [10], and minimum waiting time [11]. However, in a dynamic spectrum environment, as the pre-selected channel may no longer be available, relying on a single target channel may result in low probability of link maintenance. A better mechanism is to select multiple target channels and try each target channel sequentially. The visiting order of these target channels is crucial to spectrum handoff. In our prior work [12], we designed a target channel visiting order which can realize the minimum probability of spectrum handoff failure. In this paper, we consider spectrum handoff delay as the objective for target channel visiting order design. We propose to use particle swarm optimization (PSO) to solve this combinatorial problem. PSO is an intelligent bio-inspired optimization algorithm that has shown its effectiveness for spectrum sensing, spectrum allocation and link adaptation in cognitive radio in our prior work [13-15]. To the best of our knowledge, this paper applies PSO for target channel visiting order design for spectrum handoff for the first time. Simulations are conducted to validate the effectiveness of the proposed PSO based algorithm in determining the target channel visiting order in this paper.

The rest of the paper is organized as follows. In Section 2, we formulate the target channel visiting order design problem for spectrum handoff as a combinatorial optimization problem which aims to minimize spectrum handoff delay. In Section 3, we propose PSO for solving the problem. In Section 4, simulation results are provided and finally in Section 5 , conclusions are made.

\section{Problem Formulation}

We consider a similar system model as we formulated in [12]. Specifically, we consider two cognitive radios in a cognitive radio network communicating with each other. They operate on the same frequency range which consists of $N$ channels. During communications, the two radios exchange the channel state information so common vacant channels are known to both radios. We 
denote $M$ current vacant channels (except the current operating channel) as $\left\{c_{k} \in\{1,2, \ldots, N\}\right\}_{k=1}^{M}$, $M \leq N-1$. If a primary user appears on the current channel, the two cognitive radios would initiate spectrum handoff and switch to another vacant channel for data transmission. A proactive-sensing spectrum handoff is assumed where the target channels for spectrum handoff are ready before initiating the spectrum handoff process [16]. In this paper, $\left\{c_{k} \in\{1,2, \ldots, N\}\right\}_{k=1}^{M}$ are used as target channels for spectrum handoff. Before spectrum handoff, the visiting order of these target channels needs to be determined to optimize some objective. Denote the vector corresponding to the visiting order as $\boldsymbol{v}=\left[v_{1}, v_{2}, \ldots, v_{M}\right]$, then the process of spectrum handoff can be illustrated as in Figure 1 . When handshake on a particular channel is successful, spectrum handoff is completed successfully and a new link is maintained on this channel. However, in a dynamic spectrum environment, as primary users may reoccupy the visiting target channel during spectrum handoff, handshake may still fail. When CRs try out all the channels and all handshakes fail, then spectrum handoff is failed.

Figure 1. Handshake process for spectrum handoff.

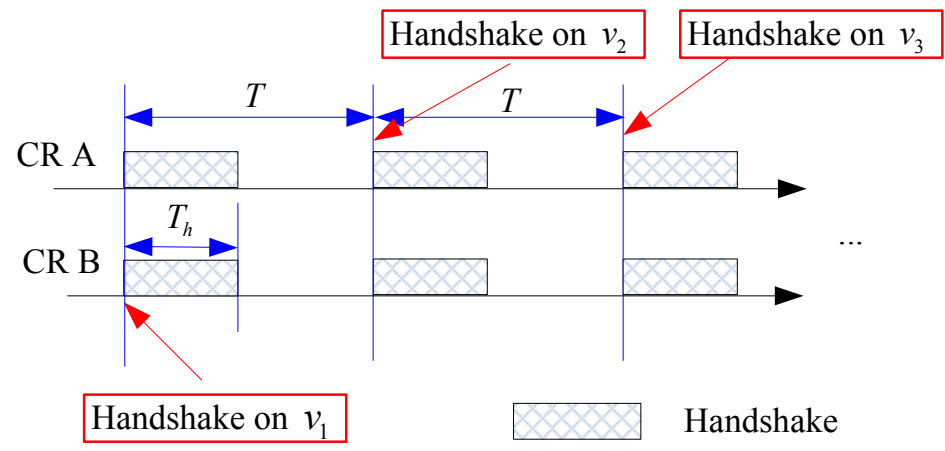

Denote $\rho_{i}$ as the probability that the handshake on $v_{i}$ is successful, then the probability of spectrum handoff failure is

$$
P_{\text {fail }}=\prod_{i=1}^{M}\left(1-\rho_{i}\right)
$$

An important metric for evaluating spectrum handoff is spectrum handoff delay. If spectrum handoff is successful, then the handoff delay is $T \ell$, where $\ell$ is the number of handshakes till success. If spectrum handoff is failed, then some other rendezvous scheme needs to be performed to maintain communications. We assume the rendezvous time is $\tau$. So the handoff delay when spectrum handoff is failed is $M T+\tau$. The expected handoff delay is

$$
E[t]=\sum_{L=1}^{M} L T \operatorname{Pr}\{\ell=L\}+(M T+\tau) P_{\text {fail }}=T p_{1}+\sum_{i=2}^{M} i T p_{i} \prod_{k=1}^{i-1}\left(1-p_{k}\right)+(M T+\tau) \prod_{i=1}^{M}\left(1-p_{i}\right)
$$

Denote the set of vectors corresponding to all permutations of all elements in $\left\{c_{k}\right\}_{k=1}^{M}$ as $\Omega$, then in order to minimize spectrum handoff delay, the problem of target channel visiting order design for spectrum handoff can be represented as

$$
\boldsymbol{v}^{*}=\underset{v \in \Omega}{\arg \min } E[t]
$$


Note that $\rho_{i}$ depends on the specific distribution of channel $v_{i}$ remaining vacant. In this paper, we consider five distributions: Uniform, Exponential [17], Generalized Pareto [18], Rayleigh [19], and Weibull [19]. As in [17], we do not consider the case where the channel state changes twice or more because the probability is too low within the relatively short duration. The probability density functions (PDFs) and the corresponding expressions of $\rho_{i}$ are shown in Table 1. For uniform distribution, the probability that the handshake on $v_{i}$ is failed equals to the probability that the state of channel $v_{i}$ changes to occupied before the end of this handshake, which is

$$
1-\rho_{i}=\left\{\begin{array}{l}
\int_{0}^{(i-1) T+T_{h}} 1 / b_{v_{i}} d x=\frac{(i-1) T+T_{h}}{b_{v_{i}}}, \quad(i-1) T+T<b_{v_{i}} \\
1, \quad(i-1) T+T \geq b_{v_{i}}
\end{array}\right.
$$

Equation (4) can be further simplified as

$$
1-\rho_{i}=\min \left\{\frac{(i-1) T+T_{h}}{b_{v_{i}}}, 1\right\}
$$

Other expressions of $\rho_{i}$ can be obtained similarly.

\begin{tabular}{|c|c|c|}
\hline Distribution & PDF & $\rho_{i}$ \\
\hline Uniform $\left(b_{v_{i}}>0\right)$ & $f(x)=\left\{\begin{array}{l}1 / b_{v_{i}}, \quad 0<x<b_{v_{i}} \\
0, \text { else. }\end{array}\right.$ & $1-\min \left\{\frac{(i-1) T+T_{h}}{b_{v_{i}}}, 1\right\}$ \\
\hline Exponential $\left(\lambda_{v_{i}}>0\right)$ & $f(x)=\left\{\begin{array}{l}\lambda_{v_{i}} e^{-\lambda_{v_{i}} x}, \quad x>0 \\
0, \text { else. }\end{array}\right.$ & $\exp \left(-\lambda_{v_{i}}\left((i-1) T+T_{h}\right)\right)$ \\
\hline $\begin{array}{l}\text { Generalized Pareto } \\
\left(\sigma_{v_{i}}>0, k \neq 0\right)\end{array}$ & $f(x)=\left\{\begin{array}{l}\frac{1}{\sigma_{v_{i}}}\left(1+k \frac{x}{\sigma_{v_{i}}}\right)^{-1-1 / k} \quad, x>0 \\
0, \text { else }\end{array}\right.$ & $\left(1+k \frac{(i-1) T+T_{h}}{\sigma_{v_{i}}}\right)^{-1 / k}$ \\
\hline Rayleigh $\left(\sigma_{v_{i}}>0\right)$ & $f(x)=\left\{\begin{array}{l}\frac{x}{\sigma_{v_{i}}^{2}} e^{-x^{2} / 2 \sigma_{v_{i}}^{2}}, x>0 \\
0, \text { else }\end{array}\right.$ & $\exp \left(-\frac{\left((i-1) T+T_{h}\right)^{2}}{2 \sigma_{v_{i}}^{2}}\right)$ \\
\hline $\operatorname{Weibull}\left(\lambda_{v_{i}}>0, \alpha>0\right)$ & $f(x)=\left\{\begin{array}{l}\alpha \lambda_{v_{i}} x^{\alpha-1} e^{-\lambda_{v_{i}} x^{\alpha}}, x>0 \\
0, \text { else }\end{array}\right.$ & $\exp \left(-\lambda_{v_{i}}\left((i-1) T+T_{h}\right)^{\alpha}\right)$ \\
\hline
\end{tabular}

Table 1. Distributions of the time duration during which a channel remains vacant.

\section{Proposed Target Channel Visiting Order Design Algorithm}

The optimal solution for combinatorial optimization problem (3) is hard to deduct. In this paper, we propose to use PSO for solving this problem. 


\subsection{Introduction of $P S O$}

PSO is a bio-inspired optimization method which is inspired by observing the bird flock [20]. In PSO, each solution is a bird in the swarm and is referred to as a "particle". In order to solve optimization problems in a discrete number space, Kennedy and Eberhart [21] developed a discrete binary version of PSO, which is the focus of this paper.

To begin with the iteration, a swarm with $S$ particles is initialized. Let $\boldsymbol{x}_{i}^{k}=\left[x_{i 1}^{k}, x_{i 2}^{k}, \ldots, x_{i D}^{k}\right]$ denote the position of particle $i(1 \leq i \leq S)$ at iteration $k$, where $D$ is the number of dimensions to represent a particle. $x_{i d}^{k}$ takes binary values from $\{0,1\}$. The velocity of particle $i$ at iteration $k$ is denoted as $\boldsymbol{y}_{i}^{k}=\left[y_{i 1}^{k}, y_{i 2}^{k}, \ldots, y_{i D}^{k}\right], \boldsymbol{y}_{i d}^{k} \in R$. Each particle in the swarm is assigned a fitness value indicating how good it is for an optimization objective. We use $\boldsymbol{p}_{i}^{k}=\left[p_{i 1}^{k}, p_{i 2}^{k}, \ldots p_{i D}^{k}\right]$ and $\boldsymbol{p}_{g}^{k}=\left[p_{g 1}^{k}, p_{g 2}^{k}, \ldots, p_{g D}^{k}\right]$ to denote the best solution that particle $i$ and the whole swarm have obtained until iteration $k$, respectively. At each iteration, each particle adjusts its velocity according to its last velocity, its distance to the best solution it has obtained and its distance to the best solution of the swarm. The velocity of the particle is updated as follows:

$$
y_{i d}^{k}=y_{i d}^{k-1}+\xi_{1} r_{1}\left(p_{i d}^{k-1}-x_{i d}^{k-1}\right)+\xi_{2} r_{2}\left(p_{g d}^{k-1}-x_{i d}^{k-1}\right)
$$

where $\xi_{1}$ and $\xi_{2}$ are two positive constants, $r_{1}$ and $r_{2}$ are random numbers uniformly chosen from the range $[0,1]$. Furthermore, the velocity is transformed to a value in the range $[0,1]$ by using the following sigmoid function:

$$
\operatorname{sig}\left(y_{i d}^{k}\right)=\frac{1}{1+\exp \left(-y_{i d}^{k}\right)}
$$

where $\operatorname{sig}\left(y_{i d}^{k}\right)$ denotes the probability of $x_{i d}^{k}$ taking 1 . According to $\operatorname{sig}\left(y_{i d}^{k}\right), x_{i d}^{k}$ can be updated as:

$$
x_{i d}^{t}= \begin{cases}1, & \text { if } r<\operatorname{sig}\left(y_{i d}^{k}\right) \\ 0, & \text { else }\end{cases}
$$

where $r$ is a random number uniformly distributed in [0,1]. In the discrete PSO, a maximum velocity $V_{\max }$ is used to avoid $\operatorname{sig}\left(y_{i d}^{k}\right)$ approaching 0 or 1, i.e., $y_{i d}^{k} \in\left[-V_{\max },+V_{\max }\right]$.

\subsection{Proposed PSO Based Algorithm for Target Channel Visiting Order Design}

The first step to use PSO for solving problem (3) is to map the particle to a possible solution. As the position of a particle contains binary bits while the visiting order takes decimal values, we need to convert these binary bits to decimal integers or the way around. Figure 2a shows the mapping process. We need $B=\left\lceil\log _{2} M\right\rceil$ bits to represent a value in $[1, M]$, where $\lceil a\rceil$ denotes the closest integer which is greater than $a$. In consequence, the number of bits in a particle position is $M B=M\left\lceil\log _{2} M\right\rceil$. Consecutive $B$ bits in the position represent a channel index. For instance, 000 represents channel $c_{1}$, 001 represents channel $c_{2}$, and so on. Figure $2 \mathrm{~b}$ illustrates an example. 
Figure 2. Mapping the position to the solution. (a) General representation. (b) An example where $M=6$ and $B=\left\lceil\log _{2} M\right\rceil=3$.

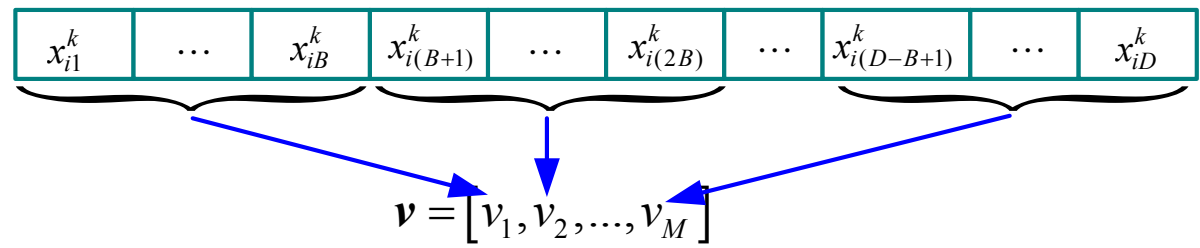

(a)

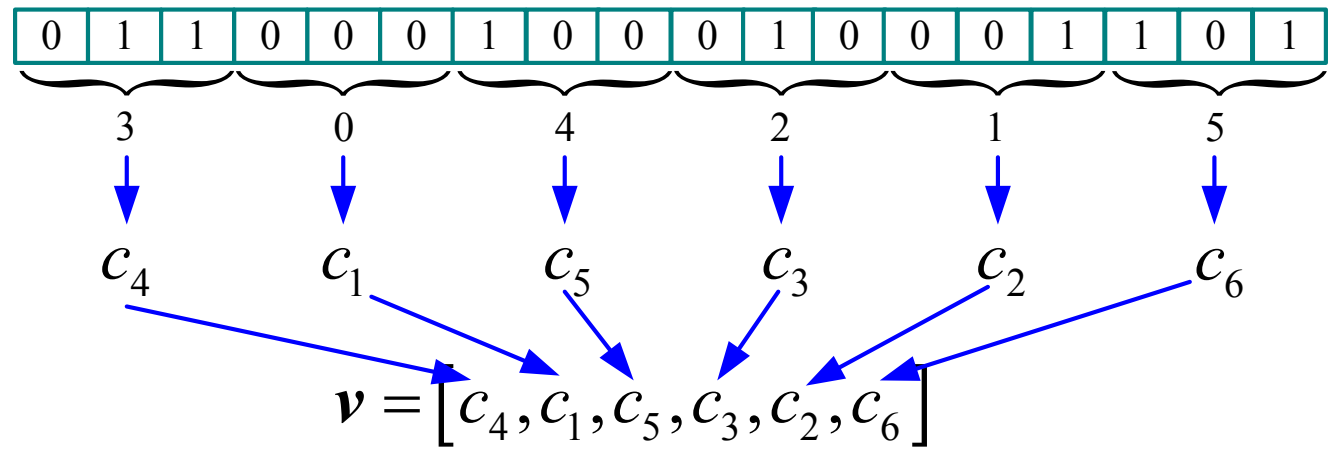

(b)

The initial swarm is generated randomly to maintain a uniform distribution of these particles on the search space. Mapping a randomly generated position to a specific $v$ may result in a solution which contains repeated channels. As a same target channel will not be visited twice or more in spectrum handoff, we propose the following procedure to ensure that a solution is valid. For position $\boldsymbol{x}_{i}^{k}=\left[x_{i 1}^{k}, x_{i 2}^{k}, \ldots, x_{i D}^{k}\right]$, we convert it to decimal integers and denote the resulting vector as $\boldsymbol{z}_{i}^{k}=\left[z_{i 1}^{k}, z_{i 2}^{k}, \ldots, z_{i M}^{k}\right]$. Update $\boldsymbol{z}_{i}^{k}$ by computing $\boldsymbol{z}_{i}^{k}=\left[z_{i 1}^{k} \bmod M, z_{i 2}^{k} \bmod M, \ldots, z_{i M}^{k} \bmod M\right]$, where $a \bmod M$ computes the reminder obtained after $a$ is divided by $M$. Store all distinct values in $\boldsymbol{z}_{i}^{k}$ on a set $\Omega$. Denote $\square=[0,1,2, \ldots, M-1]$. If the number of elements in $\Omega$ is smaller than $M$, we repeat the following two steps to make $\boldsymbol{z}_{i}^{k}$ valid: (a) For any two elements in $\boldsymbol{z}_{i}^{k}$ which are identical, randomly choose one of them and replace it with a value (denoted by $\lambda$ ) randomly chosen from the set $\Theta=\square-\Omega$; (b) update $\Omega$ by adding $\lambda$ into the set. The above procedure is repeated when all elements in $\boldsymbol{z}_{i}^{k}$ are distinct. After the above procedure, $\boldsymbol{z}_{i}^{k}$ is converted back to binary string and $\boldsymbol{x}_{i}^{k}=\left[x_{i 1}^{k}, x_{i 2}^{k}, \ldots, x_{i D}^{k}\right]$ is replaced by this string. For simplicity, we refer to this procedure as "position correction" in the rest of the paper. Fig. 3 shows an example of "position correction".

Figure 3. An example of "position correction" where $M=6$.

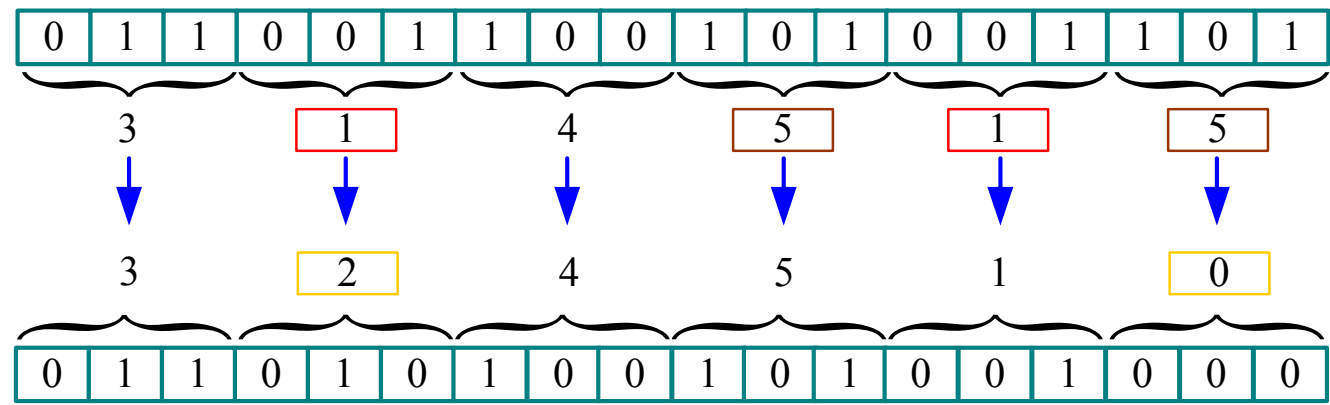


After initialization, the fitness of each particle is evaluated. We use the opposite of handoff delay (2) for fitness function. After fitness evaluation, the velocity and the position are updated by (6) and (8) and a new swarm of particle is obtained. Note that we also need to use the procedure of "position correction" discussed previously to make all positions valid. The iteration continues until the maximum number of iterations is reached. The proposed algorithm is shown in Table 2.

Table 2. Proposed algorithm for target channel visiting order.

\begin{tabular}{|c|c|}
\hline Steps & Procedures \\
\hline 1 & $\begin{array}{l}\text { Swarm initialization. Set } k=0 \text {, and randomly generate } x_{i d}^{k} \text { and } y_{i d}^{k} \text {, where } x_{i d}^{k} \in\{0,1\}, \\
y_{i d}^{k} \in\left[-V_{\max },+V_{\max }\right] \text {, and } 1 \leq i \leq S \text {. Apply "position correction" procedure to all particles in the } \\
\text { swarm. }\end{array}$ \\
\hline 2 & $\begin{array}{l}\text { Fitness evaluation. Compute the fitness value of each particle according to (2). Set } \\
\boldsymbol{p}_{i}^{k}=\left[p_{i 1}^{k}, p_{i 2}^{k}, \ldots p_{i D}^{k}\right] \text { and } \boldsymbol{p}_{g}^{k}=\left[p_{g 1}^{k}, p_{g 2}^{k}, \ldots, p_{g D}^{k}\right] \text {, where } g \text { is the index of the particle which } \\
\text { has the highest fitness value. }\end{array}$ \\
\hline 3 & $\begin{array}{l}\text { Velocity updating. Set } k=k+1 \text {, and update the velocity of the particle according to (6). If } \\
y_{i d}^{k}>V_{\max } \text {, set } y_{i d}^{k}=V_{\max } \text {; if } y_{i d}^{k}<-V_{\max } \text {, set } y_{i d}^{k}=-V_{\max } \text {. }\end{array}$ \\
\hline 4 & $\begin{array}{l}\text { Position updating. Update the position of the particle according to (7). Apply "position } \\
\text { correction" procedure to all particles in the swarm. }\end{array}$ \\
\hline 5 & $\begin{array}{l}\text { Fitness evaluation. Compute the fitness value of each particle according to (2). For particle } i \text {, if } \\
\text { it's fitness value is greater than the fitness value of } \boldsymbol{p}_{i}^{k-1} \text {, then set } \boldsymbol{p}_{i}^{k}=\boldsymbol{x}_{i}^{k} \text {; if it's fitness value is } \\
\text { greater than the fitness value of } \boldsymbol{p}_{g}^{k-1} \text {, then set } \boldsymbol{p}_{g}^{k}=\boldsymbol{x}_{i}^{k} \text {. }\end{array}$ \\
\hline 6 & $\begin{array}{l}\text { Stop criteria evaluation. If } k \text { equals to the predefined maximum iteration, the algorithm is } \\
\text { terminated; otherwise, go to step } 3 \text {. }\end{array}$ \\
\hline
\end{tabular}

\section{Simulation Results}

In the simulations, we assume $T=40 \mathrm{~ms}, T_{h}=4 \mathrm{~ms}$ and $T_{r}=400 \mathrm{~ms}$. Eight target channels are considered with the mean vacant time durations of $10 \mathrm{~ms}, 60 \mathrm{~ms}, 25 \mathrm{~ms}, 170 \mathrm{~ms}, 83 \mathrm{~ms}, 5 \mathrm{~ms}, 54 \mathrm{~ms}$, and $155 \mathrm{~ms}$, respectively. The parameters for PSO are as follows. Thirty habitats in a swarm are used. $\xi_{1}=\xi_{2}=2 . V_{\max }=4$. Figure 4 illustrates the convergence property of the algorithm. It can be observed that as the number of iterations increases, better solutions are obtained. Note that we only plot results when time duration during which a channel remains vacant follows Uniform and Exponential distributions. Similar trends have been observed with the other three distributions. For simplicity, we omitted these results.

Tables 3 and 4 show the performance of the proposed algorithm compared with the other two target channel visiting methods: the random target channel visiting and the optimal target channel visiting. Two cases are considered respectively: (Case A) Nine target channels with the mean vacant time durations of $170 \mathrm{~ms}, 30 \mathrm{~ms}, 210 \mathrm{~ms}, 300 \mathrm{~ms}, 52 \mathrm{~ms}, 5 \mathrm{~ms}, 130 \mathrm{~ms}, 59 \mathrm{~ms}, 111 \mathrm{~ms}$, respectively, and (Case B) Eight target channels with the mean vacant time durations of $10 \mathrm{~ms}, 60 \mathrm{~ms}, 25 \mathrm{~ms}, 170 \mathrm{~ms}$, $83 \mathrm{~ms}, 5 \mathrm{~ms}, 54 \mathrm{~ms}$, and $155 \mathrm{~ms}$, respectively. The optimal target channel visiting order is obtained by exhaustive search. It can be seen that the proposed method performs far better than the random channel visiting scheme. The solutions obtained by the proposed algorithm are very close to the optimal solutions. The small standard deviation values also indicate that the proposed scheme is quite stable. 
Figure 4. Convergence property of the algorithm averaged over 100 independent experiments. (a) Performance of the best particle of the swarm (time duration during which a channel remains vacant follows Uniform distribution). (b) Average performance of the whole swarm (time duration during which a channel remains vacant follows Uniform distribution). (c) Performance of the best particle of the swarm (time duration during which a channel remains vacant follows Exponential distribution). (d) Average performance of the whole swarm (time duration during which a channel remains vacant follows Exponential distribution).

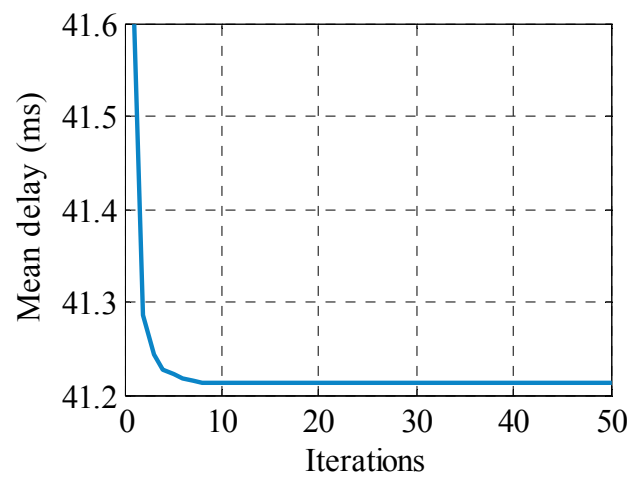

(a)

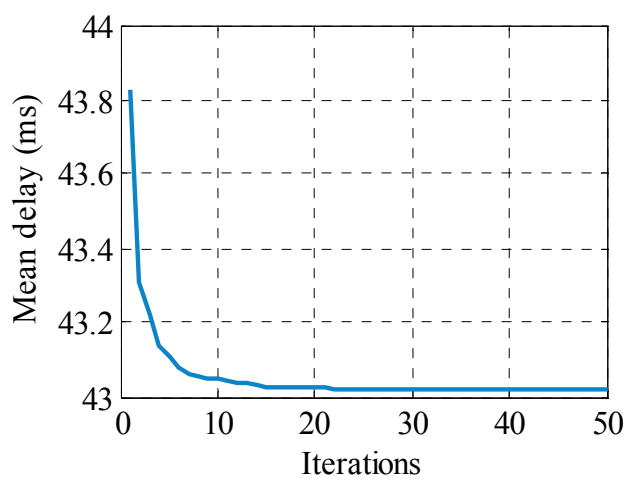

(c)

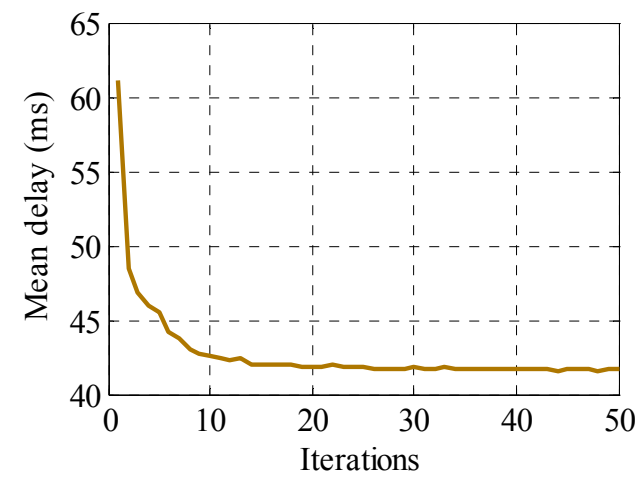

(b)

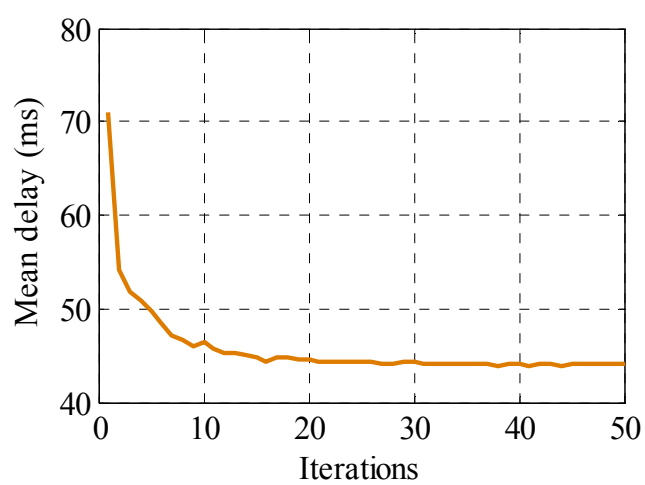

(d)

Table 3. Performance comparison (Case A). Note that "Proposed10" and "Proposed50" stands for "Proposed algorithm with 10 iterations" and "Proposed algorithm with 50 iterations", respectively.

\begin{tabular}{ccccccc}
\hline \multicolumn{2}{c}{ Distribution } & Uniform & Exponential & Pareto & Rayleigh & Weibull \\
\hline \multirow{4}{*}{ Mean } & Random & 47.1070 & 50.5568 & 43.1053 & 42.9436 & 44.4931 \\
& Optimal & 40.4067 & 40.9428 & 40.3052 & 40.0660 & 40.0651 \\
& Proposed10 & 40.4411 & 41.0199 & 40.3109 & 40.0062 & 40.0694 \\
& Proposed50 & 40.4159 & 40.9638 & 40.3063 & 40.0060 & 40.0660 \\
\hline \multirow{2}{*}{ Standard } & Random & 13.6106 & 16.2251 & 4.8572 & 8.5445 & 11.0222 \\
Deviation & Proposed10 & 0.0269 & 0.0483 & 0.0048 & $1.2614 \times 10^{-4}$ & 0.0035 \\
& Proposed50 & 0.0119 & 0.0255 & 0.0011 & $3.0954 \times 10^{-5}$ & 0.0012 \\
\hline
\end{tabular}


Table 4. Performance comparison (Case B). Note that "Proposed10" and "Proposed50" stands for "Proposed algorithm with 10 iterations" and "Proposed algorithm with 50 iterations", respectively.

\begin{tabular}{ccccccc}
\hline \multicolumn{2}{c}{ Distribution } & Uniform & Exponential & Pareto & Rayleigh & Weibull \\
\hline \multirow{4}{*}{ Mean } & Random & 60.8031 & 70.3932 & 46.3667 & 49.4641 & 54.7102 \\
& Optimal & 41.2141 & 43.0155 & 40.6554 & 40.0270 & 40.2594 \\
& Proposed10 & 41.2141 & 43.0481 & 40.6614 & 40.0270 & 40.2604 \\
& Proposed50 & 41.2141 & 43.0222 & 40.6568 & 40.0270 & 40.2595 \\
\hline \multirow{2}{*}{ Standard } & Random & 31.9083 & 35.4822 & 7.3149 & 22.8160 & 27.3215 \\
Deviation & Proposed10 & $7.1776 \times 10^{-15}$ & 0.0449 & 0.0061 & $1.9842 \times 10^{-5}$ & 0.0021 \\
& Proposed50 & $7.1776 \times 10^{-15}$ & 0.0125 & 0.0022 & $1.8236 \times 10^{-6}$ & $3.6255 \times 10^{-4}$ \\
\hline
\end{tabular}

Tables 3 and 4 show the performance of the proposed algorithm compared with the other two target channel visiting methods: the random target channel visiting and the optimal target channel visiting. Two cases are considered respectively: (Case A) Nine target channels with the mean vacant time durations of $170 \mathrm{~ms}, 30 \mathrm{~ms}, 210 \mathrm{~ms}, 300 \mathrm{~ms}, 52 \mathrm{~ms}, 5 \mathrm{~ms}, 130 \mathrm{~ms}, 59 \mathrm{~ms}, 111 \mathrm{~ms}$, respectively, and (Case B) Eight target channels with the mean vacant time durations of $10 \mathrm{~ms}, 60 \mathrm{~ms}, 25 \mathrm{~ms}, 170 \mathrm{~ms}$, $83 \mathrm{~ms}, 5 \mathrm{~ms}, 54 \mathrm{~ms}$, and $155 \mathrm{~ms}$, respectively. The optimal target channel visiting order is obtained by exhaustive search. It can be seen that the proposed method performs far better than the random channel visiting scheme. The solutions obtained by the proposed algorithm are very close to the optimal solutions. The small standard deviation values also indicate that the proposed scheme is quite stable.

\section{Conclusions}

In this paper, we have investigated the problem of designing target channel visiting order for spectrum handoff to minimize the expected spectrum handoff delay. We proposed discrete PSO for solving this combinatorial problem. Our results show that the proposed algorithm performs far better than random target channel visiting scheme in terms of the obtained mean handoff delay and the standard deviation of obtained solutions. Another attractive result is that the solutions obtained by our PSO based algorithm are very close to the optimal solutions which are obtained by exhaustive search. An interesting future work is to use some other bio-inspired optimization method such as cuckoo search [22] and bat algorithm [23] to solve the problem.

\section{Acknowledgments}

The authors thank the anonymous reviewers for their insightful comments.

\section{Author Contributions}

Xiaoniu Yang initiated the idea of this research. Shilian Zheng formulated the optimization problem, proposed the procedure of the algorithm, and wrote the initial manuscript, which is critically reviewed by Zhijin Zhao and Xiaoniu Yang. Zhijin Zhao also helped with the PSO design. Changlin Luo helped with the Matlab coding and did the simulations. 


\section{Conflicts of Interest}

The authors declare no conflict of interest.

\section{References}

1. Federal Communications Commissions. Spectrum Policy Task Force Report; ET Docket No. 02-135; Federal Communications Commissions: Washington, DC, USA, 2002.

2. Mitola, J. Cognitive radio for flexible mobile multimedia communications. In Proceedings of the 6th International Workshop on Mobile Multimedia Communications, San Diego, CA, USA, 15-17 November 1999; pp. 3-10.

3. Akyildiz, I.F.; Lee, W.-Y.; Vuran, M.C.; Mohanty, S. Next generation/dynamic spectrum access/cognitive radio wireless networks: A survey. Comput. Netw. 2006, 50, 2127-2159.

4. Wang, L.-C.; Wang, C.-W.; Chang, C.-J. Optimal target channel sequence design for multiple spectrum handoffs in cognitive radio networks. IEEE Trans. Commun. 2012, 60, 2444-2455.

5. Kim, H.; Shin, K.G. Efficient discovery of spectrum opportunities with MAC-layer sensing in cognitive radio networks. IEEE Trans. Mob. Comput. 2008, 7, 533-545.

6. Jiang, H.; Lai, L.; Fan, R.; Poor, H.V. Optimal selection of channel sensing order in cognitive radio. IEEE Trans. Wirel. Commun. 2009, 8, 297-307.

7. Wu, C.; He, C.; Jiang, L. Spectrum handoff scheme based on recommended channel sensing sequence. China Commun. 2013, 10, 18-26.

8. Yoon, S.-U.; Ekici, E. Voluntary spectrum handoff: A novel approach to spectrum management in CRNs. In Proceedings of 2010 IEEE International Conference on Communications (ICC), Cape Town, South Africa, 23-27 May 2010; pp. 1-5.

9. Zhang, W.; Yeo, C.K. Sequential sensing based spectrum handoff in cognitive radio networks with multiple users. Comput. Netw. 2014, 58, 87-98.

10. Song, Y.; Xie, J. ProSpect: A proactive spectrum handoff framework for cognitive radio ad hoc networks without common control channel. IEEE Trans. Mob. Comput. 2012, 11, 1127-1139.

11. Oo, T.Z.; Hong, C.S.; Lee, S. Alternating renewal framework for estimation in spectrum sensing policy and proactive spectrum handoff. In Proceedings of International Conference on Information Networking, Bangkok, Thailand, 28-30 January 2013; pp. 330-335.

12. Zheng, S.; Yang, X.; Chen, S.; Lou, C. Target channel sequence selection scheme for proactive-decision spectrum handoff. IEEE Commun. Lett. 2011, 15, 1332-1334.

13. Zhao, Z.; Peng, Z.; Zheng, S.; Shang, J. Cognitive radio spectrum allocation using evolutionary algorithms. IEEE Trans. Wirel. Commun. 2009, 8, 4421-4425.

14. Zhao, Z.; Xu, S.; Zheng, S.; Shang, J. Cognitive radio adaptation using particle swarm optimization. Wirel. Commun. Mob. Comput. 2009, 9, 875-881.

15. Zheng, S.; Lou, C.; Yang, X. Cooperative spectrum sensing using particle swarm optimization. Electron. Lett. 2010, 46, 1525-1526.

16. Wang, L.-C.; Wang, C.-W.; Chang, C.-J. Modeling and analysis for spectrum handoff in cognitive radio networks. IEEE Trans. Mob. Comput. 2012, 11, 1499-1513. 
17. Zhou, X.; Li, Y.; Kwon, Y.H.; Soong, A.C.K. Detection timing and channel selection for periodic spectrum sensing in cognitive radio. In Proceedings of IEEE Global Telecommunications Conference, New Orleans, LO, USA, 30 November-4 December 2008; pp. 1-5.

18. Geirhofer, S.; Tong, L.; Sadler, B.M. A measurement-based model for dynamic spectrum access in WLAN channels. In Proceedings of IEEE Military Conference, Washington, DC, USA, 23-25 October 2006; pp. 1-7.

19. Pawelczak, P.; Pollin, S.; So, H.-S.; Motamedi, A.; Bahai, A.; Prasad, R.V.; Hekmat, R. State of the art in opportunistic spectrum access medium access control design. In Proceedings of 3rd International Conference on Cognitive Radio Oriented Wireless Networks and Communications, Singapore, 15-17 May 2008; pp. 1-6.

20. Kennedy, J.; Eberhart, R. Particle swarm optimization. In Proceedings of IEEE International Conference on Neural Networks, Perth, Australia, 27 November-01 December 1995; pp. 1942-1948.

21. Kennedy, J.; Eberhart, R. A discrete binary version of the particle swarm algorithm. In Proceedings of the Conference on Systems, Man, and Cybernetics, Orlando, FL, USA, 12-15 October 1997; pp. 4104-4109.

22. Yang, X.S.; Deb, S. Engineering optimisation by cuckoo search. Int. J. Math. Model. Numer. Optim. 2010, 1, 330-343.

23. Yang, X.S.; Gandomi, A.H. Bat algorithm: A novel approach for global engineering optimization. Eng. Comput. 2012, 29, 464-483.

(C) 2014 by the authors; licensee MDPI, Basel, Switzerland. This article is an open access article distributed under the terms and conditions of the Creative Commons Attribution license (http://creativecommons.org/licenses/by/3.0/). 